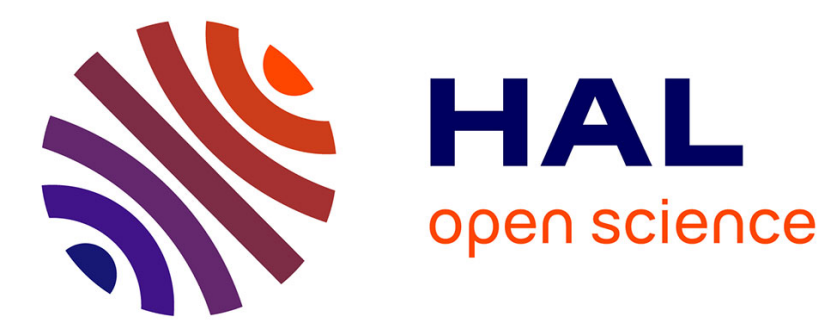

\title{
Effect of mineral surface properties (alumina, kaolinite) on the sorptive fractionation mechanisms of soil fulvic acids: Molecular-scale ESI-MS studies
}

\author{
Guillaume Fleury, Mirella del Nero, Rémi Barillon
}

\section{To cite this version:}

Guillaume Fleury, Mirella del Nero, Rémi Barillon. Effect of mineral surface properties (alumina, kaolinite) on the sorptive fractionation mechanisms of soil fulvic acids: Molecular-scale ESI-MS studies. Geochimica et Cosmochimica Acta, 2017, 196, pp.1 - 17. 10.1016/j.gca.2016.09.029 . hal-01468584

\author{
HAL Id: hal-01468584 \\ https://hal.science/hal-01468584
}

Submitted on 10 Feb 2022

HAL is a multi-disciplinary open access archive for the deposit and dissemination of scientific research documents, whether they are published or not. The documents may come from teaching and research institutions in France or abroad, or from public or private research centers.
L'archive ouverte pluridisciplinaire HAL, est destinée au dépôt et à la diffusion de documents scientifiques de niveau recherche, publiés ou non, émanant des établissements d'enseignement et de recherche français ou étrangers, des laboratoires publics ou privés. 
8 Institut Pluridisciplinaire Hubert Curien, UMR7178 Université de Strasbourg / CNRS,

923 rue du Loess, 67037 Strasbourg, France 


\section{ABSTRACT}

26 We addressed the effects of mineral surface properties (kaolinite versus Al-oxide) on the sorption-driven 27 fractionation of a soil fulvic acid (FA) at acidic $\mathrm{pH}$, mainly by means of ESI(-)-FTMS analysis of initial and 28 supernatant solutions of FA sorption batch experiments. The MS data provided clear molecular-scale evidence 29 of distinct mechanisms and molecular parameters controlling the FA fractionation upon its sorption on clay 30 and oxide surfaces, respectively.

31 Identification of sorbing and not-sorbing FA compounds in kaolinite-solution systems revealed a weak 32 fractionation amongst members of $-\mathrm{CO}_{2}$ series of aliphatics or not-condensed aromatics (NCAs) at $\mathrm{pH} 3.8$, 33 and almost no sorption of poorly-oxygenated polycyclic aromatic compounds (PACs) and NCAs. This first 34 molecular-scale description of a FA fractionation in a clay-solution system suggests that H-bonding with low 35 affinity sites (aluminol/silanol) on the basal planes of the clay particles is the main mechanism of sorption.

36 Due to the predominance of such weak and poorly-selective mechanism, the sorption of aliphatic and NCA 37 molecules bearing oxygenated functionalities was prevented at $\mathrm{pH} 5$, due to dissolved $\mathrm{Al}$ competing 38 successfully for their coordination.

39 In contrast, a strong FA fractionation was observed onto alumina, with a preferential retention of PACs and 40 highly-oxygenated aliphatics and NCAs. The major part of the poorly oxygenated aliphatics was left in 41 solution. The sorption degree of NCAs and aliphatics was strongly correlated with molecular acidity. For 42 PACs and poorly-oxygenated NCAs, the sorption was driven by reactions of surface ligand exchange (for the 43 most oxygenated compounds) or by hydrophobic interactions (for the least oxygenated compounds).

45 Key words: humic substances; kaolinite; alumina; fractionation; mass spectrometry 
49 Humic substances (HSs) like fulvic acids (FAs) are complex heterogeneous mixtures of thousands of 50 biochemically stable polymeric substances (Aiken et al., 1985) which are ubiquitous in all terrestrial surface 51 systems and which account for a great part of organic carbon in soils. HSs show a variety in the composition, structure and reactivity of their constituents and functional groups (Swift, 1989; Plancque et al., 2001). It has long been known that HSs have the ability to interact with metals (Dobbs et al., 1989; Tipping, 2002; Milne et al., 2003; Janot et al., 2013), organic pollutants (Rebhun et al., 1996) and surfaces of (nano) particles of clays and Al- or Fe-oxyhydroxides (Ochs et al., 1994; Schlautman and Morgan, 1994; Feng et al., 2005; Janot et al., 2013), with the sorption of HSs resulting in its chemical fractionation between minerals and solution (Meier et al., 1999; Zhou et al., 2001; Reiller et al., 2006). Sorption-driven fractionation of HSs is expected to influence surface characteristics (reactivity, sorption capacity, charge) of minerals, dispersion behavior of suspended nanoparticles which are potential pollutants' carriers, and metal binding capacity of dissolved organic matter in many geochemical systems (Ochs et al., 1994; Schlautman and Morgan, 1994; Yang et al., 2013). Understanding of the fractionation of HS during the sorption at surfaces of mineral particles is thus of interest in many environmental issues including sorption / mobility of trace metal elements in soils and aquatic systems. It has some implications on the evaluation of the impact on health of the release of pollutants in the ecosystems and on the safety assessments of remediation processes of polluted soils or radioactive waste repositories in geological formations (Loffredo and Senesi, 2006; Bryan et al., 2012).

Building a comprehensive description of the coupled cycles of organic matter and pollutants in natural systems requires some basic knowledge of the fractionation trends exhibited by HSs during their sorption at mineral surfaces relevant to soils and waters, as well as a thorough understanding of the mechanisms and molecular parameters driving the fractionation -which is still lacking-. Speaking in a general way, several mechanisms of sorption of HSs onto minerals were proposed, including surface ligand exchange, specific adsorption, electrostatic interactions, hydrophobic effects, H-bonding and metal-ion bridging (Ochs et al., 1994; Schlautman and Morgan, 1994). In many studies, HSs were considered as bulk materials bearing polar functionalities and spectroscopic/modeling evidence was given that carboxyl functional groups of HS (Fu et al., 2005; Ghosh et al., 2009) are involved in the macroscopic sorption of HS at surfaces of iron or aluminum oxide minerals and that the contribution of phenol groups increases with pH (Filius et al., 2003; Claret et al., 
2008). In the last two decades, there has also been a growing interest in getting a detailed understanding of the behavior of HSs during their sorption at (nano) mineral - solution interfaces. Many spectroscopic evidences were provided that a strong sorptive fractionation takes place between the diverse classes of organic compounds of a humic substance (e.g. Meier et al., 1999; Claret et al., 2008; Ghosh et al., 2009). It was reported that the hydrophobic fractions of FAs and/or the organic molecules with high contents in aromatic moieties activated by oxygenated functionalities were preferentially sorbed -as compared to aliphatic fractions- on the surfaces of metallic oxide minerals (Meier et al., 1999; Zhou et al., 2001; Kaiser, 2003; Claret et al., 2008). Moreover, several studies combining size-exclusion-chromatography and spectroscopy analysis have revealed a size fractionation of fulvic or humic acids during their sorption at several mineral surfaces (e.g. Hur and Schlautman, 2004), which was explained by structural trends in the molecular weight fractions (degree of aromaticity, functionalities...) and the underlying sorption processes. Since recently, the development of an advanced technique, namely ultra-high resolution Fourier transform mass spectrometry (FTMS), has offered unique opportunities for identifying the molecules constitutive of a HA or FA (e.g. Kujawinski et al., 2002; D'Andrilli et al., 2010) and for approaching the issue of the HS-metallic oxidesolution interactions at the molecular scale (Reiller et al., 2006; Galindo and Del Nero, 2014; Galindo and Del Nero, 2015). Published FTMS data for the characterization of HSs (Plancque et al., 2001; Stenson et al., 2002; Sutton and Sposito, 2005) have supported the model proposed by Wershaw (1993) and by Engebretson and von Wandruszka (1994), and recently revived by Piccolo (2001) and Plancque et al. (2001), which describes an HS as a supramolecular association of small organic entities ("building blocks" of ca. 500 Da for FAs) assembled via weak bonds. This model has led to view an HS as a complex mixture of thousands of polycarboxylates of distinct molecular weight, degree of aromaticity or acidity, and chemical reactivity. Recently, the ESI-FTMS technique was applied by our research team to the identification of sorbing (and not sorbing) molecules of an aquatic fulvic acid (Galindo and Del Nero, 2014), and of a terrestrial humic acid (Galindo and Del Nero, 2015) during experiments of HS-alumina-solution interactions. A striking result was an inverse correlation existing between the degree of sorption of a molecule within a $\mathrm{CH} 2$ series and its number of $\mathrm{CH} 2$ groups, and a positive correlation between the degree of sorption and the number of $\mathrm{CO} 2$ groups in a $\mathrm{COO}$ series, for homologous series containing up to nine members. In agreement with previous experimental work by Evanko and Dzombak (1998) on simple organic acids and modelling work by Janot et al. (2012) suggesting that enhanced sorption with increasing number of carboxyl groups was due to changes in 
compound acidity, the correlations observed for FA by ESI-FTMS provided for the first time a molecular

106 scale evidence that the sorptive fractionation of complex mixtures of thousands of organic compounds as 107 fulvic acids was controlled by molecule acidity, which was remarkable. These ESI-FTMS findings also 108 strongly supported that the FA subunits, held together by hydrophobic forces and hydrogen bonding, were 109 disrupted during sorption because of strong FA-alumina surface interactions via surface ligand exchange 110 mechanism, which was consistent with the concept of supramolecular assembly for HS (Piccolo, 2001; 111 Piccolo et al., 2010). Hydrophobic interactions were moreover found to be a mechanism cooperating to the 112 sorption onto alumina of the poorly-oxygenated polycyclic aromatic compounds (PACs) of a terrestrial HA, 113 with the degree of molecule hydrophobicity influencing the degree of molecule sorption.

114 In contrast to the metallic oxide systems, very few information is available on the sorption-driven 115 fractionation of HSs onto clay minerals and no molecular scale description of the process has been reported so 116 far. Unlike for iron or aluminum (hydr)oxides, the aliphatic fractions of a humic acid were found to be more 117 prone to sorption on the surface of kaolinite and montmorillonite than the aromatic fractions (Wang and Xing, 118 2005; Ghosh et al., 2009). Such contrasting results between metallic oxides and clays have pointed the need to 119 acquire detailed knowledge of the molecular characteristics and the sorption mechanisms driving HS sorptive 120 fractionation in clay mineral - solution systems, which are relevant to many geochemical systems. In 121 particular, such knowledge is critically needed for developing a comprehensive understanding of the sorptive 122 fractionation of FAs in the various types of soils (rich in metal oxi-hydroxides or in clays).

123 In this study, we addressed the effects of mineral surface properties, i.e., clay versus aluminum oxide, on the 124 molecular scale fractionation of a terrestrial FA during its sorption at mineral surface by mean of 125 complementary techniques, traditional batch experiments, electro-kinetic measurements and electrospray 126 ionization FTMS. The systems under investigation included an extracted FA (PPH) and kaolinite or alumina 127 as a model sorbent taken as being representative of Al (hydr)oxides or clays in soils, respectively. First, batch sorption experiments and on-line electrophoretic mobility measurements were performed in PPH-containing mineral suspensions for studying the pH-dependency of sorption of PPH onto alumina and kaolinite and for gaining insights into surface charges of PPH-loaded particles. The use of a LTQ Orbitrap XL hybrid mass spectrometer allowed deriving the exact elemental composition of the molecules of PPH present in the solution before and after contact with the metal oxide or the clay mineral, for experiments performed under acidic and middle acidic solutions ( $\mathrm{pH} 3.5$ and 5.0, respectively). The molecular approach was used to retrieve 
the distinct orders of relative affinity of the FA molecules for the surface of a clay and a metallic oxide, respectively, and to explore the relations existing between chemical characteristics of the PPH molecules (such as number of $\mathrm{CO} 2$ functionalities, degree of aromaticity or acidity...) and their degree of relative sorption onto mineral. The molecular-scale results presented here represent a significant contribution to our understanding of the fractionation trends of a terrestrial FA during its sorption onto a clay mineral, and of the sorption mechanisms involved, and highlight the strong effects of mineral surface properties on the fractionation behavior of terrestrial fulvic acids.

\section{EXPERIMENTAL SECTION}

\subsection{Materials}

The fulvic acid denoted as "PPH" was extracted from a soil sample collected in a beech forest developed on sandstone, near La Petite-Pierre, Bas-Rhin, France. The extraction was carried out by using the International 148 Humic Substances Society (IHSS, St. Paul, MN, USA) standard method for isolation of soil fulvic acids. This method, excerpted from Sparks et al. (1996), uses XAD-8 resin adsorption to isolate such acids. Due to the chemical properties of the resin, the fulvic acids used here only contain hydrophobic organic compounds. Stock solutions of PPH fulvic acid were prepared and stored frozen in the dark until used.

Alumina colloids were Alfa Aesar's $\alpha-\mathrm{Al}_{2} \mathrm{O}_{3}$ crystallites (chemical purity: 99.95\%, surface area: $7.6 \mathrm{~m}^{2} / \mathrm{g}$ ) with a particle size of $280 \mathrm{~nm}$ as determined by dynamic light scattering $\left(\left[\alpha-\mathrm{Al}_{2} \mathrm{O}_{3}\right]=2.5 \mathrm{~g} . \mathrm{L}^{-1}\right.$, particle size determined in $\%$ of intensity, $\mathrm{w}_{50 \%}=90 \mathrm{~nm}$, polydispersity index $=0.12$ ). The kaolinite sample used was the standard sample KGa-1b provided by The Clay Minerals Society. This sample was characterized by Pruett and Webb (1993), who reported a particle distribution of $57.8 \%<2 \mu \mathrm{m}$ and $32.0 \%<0.5 \mu \mathrm{m}$, as well as a surface area of $11.7 \mathrm{~m}^{2} \cdot \mathrm{g}^{-1}$. This value for surface area of kaolinite has been used as such and no attempt was made to determine again this value.

\subsection{Experimental setup}


Experiments of FA sorption at acidic pH and 298K on alumina and kaolinite were carried out for analysis of

164 the supernatants by ESI(-)-FTMS and study of the mineral surface characteristics on FA sorptive 165 fractionation. The experiments were carried out at $\mathrm{pH} 3.8 \pm 0.1$ and $4.9 \pm 0.1$ for both minerals.

166 Alumina and kaolinite suspensions were prepared in individual PEHD tubes with solid-to-solution ratios of 25 167 and 15 g.L $\mathrm{L}^{-1}$, respectively. These values of solid-to-solution ratios were chosen so that the surface areas of alumina and kaolinite used in the experiments fall in the same range of values (ca. 170 and $150 \mathrm{~m}^{2} / \mathrm{L}$, respectively). Efforts were made to adjust the concentration of PPH added in the individual tubes, so that the values of FA-to-mineral ratios $(\mathrm{r})$ of all experiments fall within a narrow range $(\mathrm{r} \sim 5 \mathrm{mg} \mathrm{C} / \mathrm{g}$, or more precisely, $\mathrm{r} \sim 0.6$ and $0.8 \mathrm{mgC} / \mathrm{m}^{2}$ of mineral for sorption of FAs on kaolinite and alumina, respectively). No attempt was made to fix the ionic strength of the initial experimental solutions, as background salts were previously observed to alter considerably the ESI-MS response. After shaking of the tubes for 24 hours at $298 \mathrm{~K}$, the final $\mathrm{pH}$ values of the suspensions were measured. The suspensions were then centrifuged at 8000 rpm during 2.5 hours for solution-colloid separation and the supernatants were collected. Aliquots were taken for measurements of dissolved organic carbon, DOC, using a Shimadzu TOC $-\mathrm{V}_{\mathrm{CPH}}$ analyzer, and of dissolved aluminum content using Inductively Coupled Plasma Mass Spectrometry (quantification limit: $5.3 \mu \mathrm{g} / \mathrm{L}$ ). All the remaining supernatant solutions were adjusted (using a $0.01 \mathrm{M} \mathrm{HCl}$ solution) to a same $\mathrm{pH}$ value of $3.8 \pm 0.1$ prior to ESI-MS analysis. Such an acidification procedure allows a direct comparison of the datasets obtained for the different experiments (as pH is known to affect ionization of compounds during ESIMS analysis). An exception was however made for the supernatant of the PPH-kaolinite sorption experiment at $\mathrm{pH}$ 5.0, which was analyzed as collected because the ESI(-)MS spectra obtained for the acidified supernatant sub-sample were found to be of very poor quality due to the amount of salt introduced in the sample to obtain a $\mathrm{pH}$ of 3.8. It is to be noted that the ESI-MS data obtained from an analysis at $\mathrm{pH} 5.0$ cannot be straightforwardly compared to those obtained from analysis at $\mathrm{pH} 3.8$.

Complementary series of batch experiments were performed to study the macroscopic sorption of FA as a function of $\mathrm{pH}$ and to perform on-line electro-kinetic measurements of the FA-containing suspensions of kaolinite or alumina. Same procedure as that described above was used, except that an aliquot of sample was taken before centrifugation for particle electrophoretic mobility (EM) measurements, which were carried out using dynamic light scattering with a Malvern Zetasizer Nano ZS instrument. Values reported for EM correspond to mean values calculated on the basis of three replicates of a same measurement. Sorption 
isotherms and EM values in the $\mathrm{pH}$ range 3.5-7 were determined for the alumina $-\mathrm{PPH}$ systems $(\mathrm{r}=5-10$ $\mathrm{mgC} / \mathrm{g}$ ). For the kaolinite - PPH system, both the effect of $\mathrm{pH}$ and ratio $\mathrm{r}$ on the percent of FA sorption and

194 EM of particles were investigated ( $\mathrm{r}$ value up to $50 \mathrm{mgC} / \mathrm{g}$ ).

\subsection{ESI-MS instrumentation and data analysis}

\subsubsection{Instrumentation and acquisition parameters}

A Thermo Scientific LTQ Orbitrap hybrid mass spectrometer was used for the analysis in ESI negative ionization mode of native FA solutions and supernatants. Negative ion ESI appears to be the most appropriate ionization mode for the analysis of anionic compounds such as fulvic acids, which are already partly preionized in solution. Ultra-pure water was used as the electrospray solvent as other solvents have been found to alter the FA properties (Piccolo et al., 2001). The samples were infused directly into the ESI source with a spray voltage of $3.5 \mathrm{kV}$ at a flow rate of $10 \mu \mathrm{L} \cdot \mathrm{min}^{-1}$, and were analyzed in negative ion mode. Nitrogen was used as the drying and spraying gas. For each acquisition, 100 scans (2s/scan) were co-added using Xcalibur software. The Orbitrap MS was externally calibrated in negative ion mode on the 50-2000 m/z range using a LTQ/FT-Hybrid ESI Calmix solution, for a mass accuracy better than 3 ppm. Instrument operating parameters (capillary voltage and temperature, tube lens voltage) were optimized one after the other for an optimal transmission of higher $\mathrm{m} / \mathrm{z}$ ions. It was found that modifying the instrumental parameters did not affect important characteristics of spectra such as ion distribution, charge state or mass spacing patterns. The source capillary was finally held at $-50 \mathrm{~V}$ and $275^{\circ} \mathrm{C}$, and a voltage of $-240 \mathrm{~V}$ was applied to the tube lens.

213 All ESI(-) mass spectra were recorded in the ranges $120-400 \mathrm{~m} / \mathrm{z}$ and $400-798 \mathrm{~m} / \mathrm{z}$ in order to improve the 214 mass accuracy in the region of low masses as well as the signal-to-noise ratio ( $\mathrm{S} / \mathrm{N})$ for compounds having the 215 highest values of $\mathrm{m} / \mathrm{z}$ in samples.

\subsubsection{Elemental formula determination}

219 Elemental formulae were assigned to the measured $\mathrm{m} / \mathrm{z}$ values using the Xcalibur software. The following 220 criteria were chosen for assignment of molecular formulas to the peaks detected on the mass spectra: 
- Signal-to-noise ratio of peak $>4$,

- Mass accuracy better than 3 ppm,

- Application of the following ranges: ${ }^{14} \mathrm{~N} \leq 1 ;{ }^{16} \mathrm{O} \leq 50 ;{ }^{12} \mathrm{C} \leq 200 ;{ }^{1} \mathrm{H} \leq 600(\mathrm{~N}, \mathrm{O}, \mathrm{C}$ and $\mathrm{H}$ being respectively the number of Nitrogen, Oxygen, Carbon and Hydrogen atoms).

228 Elemental analyses showed that the PPH sample contains low amounts of Nitrogen $(<3 \% \mathrm{w} / \mathrm{w})$. Sulfur and

Phosphorus contents were assumed negligible $(<1 \% \mathrm{w} / \mathrm{w})$. Odd $\mathrm{m} / \mathrm{z}$ values correspond to even mass, and relate to chemical formulae with even number of nitrogen atoms. As the nitrogen content in the sample does not exceed a few percent, we assumed that compounds peaking at odd $\mathrm{m} / \mathrm{z}$ primarily contain $\mathrm{C}, \mathrm{H}$ and $\mathrm{O}$ atoms. All possible formula attributable to a given odd $\mathrm{m} / \mathrm{z}$ value were thus calculated by considering only $\mathrm{C}$, $\mathrm{H}$ and $\mathrm{O}$ atoms. Due to low nitrogen content in HS, species peaking at even $\mathrm{m} / \mathrm{z}$ were considered to contain at most one $\mathrm{N}$ atom, in addition to $\mathrm{C}, \mathrm{H}$ and $\mathrm{O}$ atoms. Ions corresponding to ${ }^{13} \mathrm{C}$ isotopes and elemental formulae that did not match the above criteria were not taken into account.

\subsubsection{Van krevelen diagrams}

The Van Krevelen (VK) diagram plots the $\mathrm{H} / \mathrm{C}$ ratio vs. the $\mathrm{O} / \mathrm{C}$ ratio of a molecule and is an effective 240 graphical method for displaying complex data from NOM analysis by ultrahigh-resolution mass spectrometry 241 (Kim et al., 2003). On the VK diagrams, the identified compounds are classified into three categories on basis 242 of their value of Aromaticity Index (A.I.) proposed by Koch and Dittmar (2006). The authors proposed a modified formula for calculating A.I., considering that half of the oxygen is $\sigma$-bound:

Aromaticity Index (A. I. ) $=\frac{1+\mathrm{C}-\frac{\mathrm{O}}{2}-\frac{\mathrm{H}}{2}}{\mathrm{C}-\frac{\mathrm{O}}{2}-\mathrm{N}}$

244 They defined two threshold values of AI to distinguish between three categories of compounds, i.e., between 245 compounds having an unambiguous polycyclic aromatic structure (PACs, having 0.67 < A.I.), a not246 condensed aromatic structure (NCAs, having $0.5<$ A.I. $<0.67$ ), and a more or less pronounced aliphatic 


\subsubsection{Definition of I}

251 In order to explore the mechanisms of the sorptive fractionation of FAs, we used the parameter I defined by 252 Galindo and Del Nero (2014). The value of I for a given ion is equal to the ratio of the normalized peak intensity of this ion in the supernatant (after sorption) on the normalized peak intensity of the ion in the initial solution (before sorption), with the normalization being made to the sum of the intensities of all the ions (Total ion current, TIC) of the considered solution. Trends in the values of I for compounds in a FA supply information on trends existing in the orders of relative affinity of compounds for the mineral surface: the lower the value of I of a FA ion, the higher its relative affinity (by comparison with that of the other detected FA ions) for the surface of the mineral. Ions with $I=0$ are totally sorbed at the surface of mineral, and ions with $\mathrm{I} \neq 0$ are distributed between the surface and the solution.

It is well known that the intensity of an ion on a mass spectrum not only depends on its concentration but also on its ionization efficiency and on the matrix, i.e. on the number, the quantity and the structure of the other ions present in the sample. A simple dilution of initial FA solution can thus possibly have an effect on the relative peak intensities of the ions in the solution. As bulk concentration of PPH is lower in the supernatants collected at the end of the sorption experiments than in the native PPH solution (due to PPH sorption at the surface of the minerals), it is necessary to determine the effect of such a sorption-driven decrease in solution concentration on the I values of the FA ions and to differentiate it from the effect on I of the sorptive fractionation process itself. To this end, we compared the ESI(-)MS spectra recorded for initial PPH solution at $\mathrm{pH} 3.8$ and at $\mathrm{pH} 4.9$ used in the experiments to those obtained for PPH native solutions diluted by a factor 2, and we calculated the "I values" of the FA ions resulting from a pure dilution of samples. Dilution by a factor of 2 was chosen because approximately $50 \%$ of total organic carbon was found to be removed from solution during the experiments of PPH sorption onto alumina and kaolinite. It was found (cf. Fig. A1 in the Appendix) that all PPH ions display I values equal to $0.85 \pm 0.15$ due to dilution effect only (except for the $400-798 \mathrm{~m} / \mathrm{z}$ range at $\mathrm{pH} 3.8$ where the values were equal to $1.05 \pm 0.15)$. Applying these results of the dilution tests to the interpretation of the I values of ions derived from the sorption experiments, it can be 
good sorption of an ion on the mineral surface, while ions showing values comprised between 0.7 and 1 (or 0.9 and 1.2 for the range $400-798 \mathrm{~m} / \mathrm{z}$ range at $\mathrm{pH} 3.8$ ) are poorly sorbed or not sorbed at all, and ions showing values higher than 1 (or 1.2) are not sorbed at all during the experiment.

\section{RESULTS}

\subsection{Macroscopic description of the FA-mineral systems}

Fig. 1 shows the percentage of organic carbon of $\mathrm{PPH}$ sorbed onto alumina or kaolinite as a function of $\mathrm{pH}$, for different FA/mineral ratios (r). The results are as follows.

Regarding alumina, first, the sorption isotherm obtained for PPH indicate a slight pH-dependency of the sorption, i.e., a slight decrease of the percentage of sorption with increasing $\mathrm{pH}$. Such a decrease with increasing $\mathrm{pH}$ value is consistent with an increase of the rate of deprotonation of both the reactive groups of the FA molecules and the aluminol sites on the surface of alumina. It has been widely reported that the carboxyl and phenol functional groups of FAs exhibit wide distributions of pK values, which are mostly lower than 4 for carboxyl groups (e.g. Ritchie and Perdue, 2003) and higher than 8 for phenol moieties (e.g. Gu et al., 1995). Moreover, the IEP of the alumina particles used here was previously found to be equal to 8.5 (Del Nero et al., 2010). An increase of the deprotonation with $\mathrm{pH}$ thus has the effect of diminishing electrostatic attractions between aluminol sites on the mineral surface and FA molecules, which is unfavorable to a close approach of the organic molecules to the vicinity of the surface. Thus, such features may lead to a slight decrease of the percentage of macroscopic sorption with $\mathrm{pH}$ observable in Fig. 1. The results of EM measurements performed on the systems investigated are presented on Fig. 2. The data show a sign reversal of the surface charge of the suspended alumina particles in the presence of $\mathrm{PPH}$, evidencing that the FA-alumina interactions are not of electrostatic nature only but involve the formation of inner-sphere fulvate complexes on the surface of alumina. Chemical sorption is indeed the only sorption process capable of inducing such a sign reversal (Fairhurst and Warwick, 1998).

Fig. 1 shows that PPH has a lower overall affinity for the kaolinite surface than for the alumina surface (cf. data at $\mathrm{r}=5 \mathrm{mgC} / \mathrm{g}$ ). There also appeared that the percentage of PPH sorption decreases with an increase of the $\mathrm{PPH} /$ kaolinite ratio (cf. data at $\mathrm{r}=5$ and $55 \mathrm{mgC} / \mathrm{g}$ ). Such a feature indicates that multiple sites on the mineral 
surface were involved in the sorption of the PPH molecules and/or several types of surface complexes of very

306 distinct values of formation constant were formed. The former hypothesis is consistent with results of many

307 previously published studies reporting the existence of weak surface sites at basal planes of kaolinite particles 308 and of strong sites (high-affinity sites) at edges and/or defects of kaolinite(Sverjensky and Sahai, 1996). The 309 high-affinity sites existing in limited amounts onto the kaolinite particles might be saturated in our 310 experiments at low surface loadings of FA. High-affinity sites have long been reported to be protonated under 311 acidic and neutral $\mathrm{pH}$ conditions whereas the early deprotonation of basal aluminol sites (Wieland and 312 Stumm, 1992) is (partly) responsible for an overall negative surface charge of kaolinite (e.g. data on Fig. 2 313 obtained in the absence of FA). EM measurements reported in Fig. 2 shows that the sorption of the PPH 314 molecules imparts negative charges to the kaolinite surface, which is consistent with involvement of the high315 affinity sites in the sorption.

316 In summary, surface properties of mineral have an effect on the macroscopic behavior of sorption of FA at 317 acidic/near neutral $\mathrm{pH}$, which highlights a need for further understanding their effect on the fractionation of 318 FA.

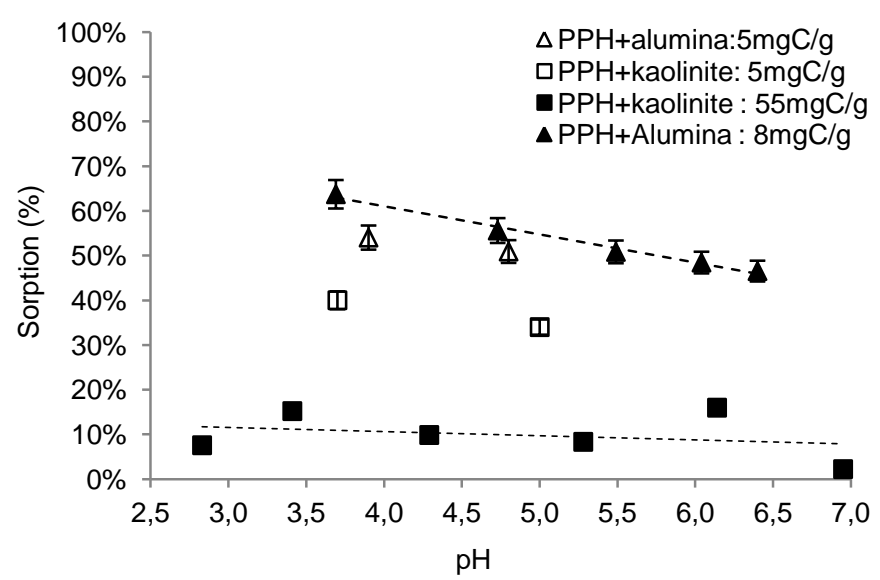

321 Fig. 1: Sorption isotherms of organic carbon obtained for different PPH-to-mineral ratios in alumina or 322 kaolinite suspensions. 


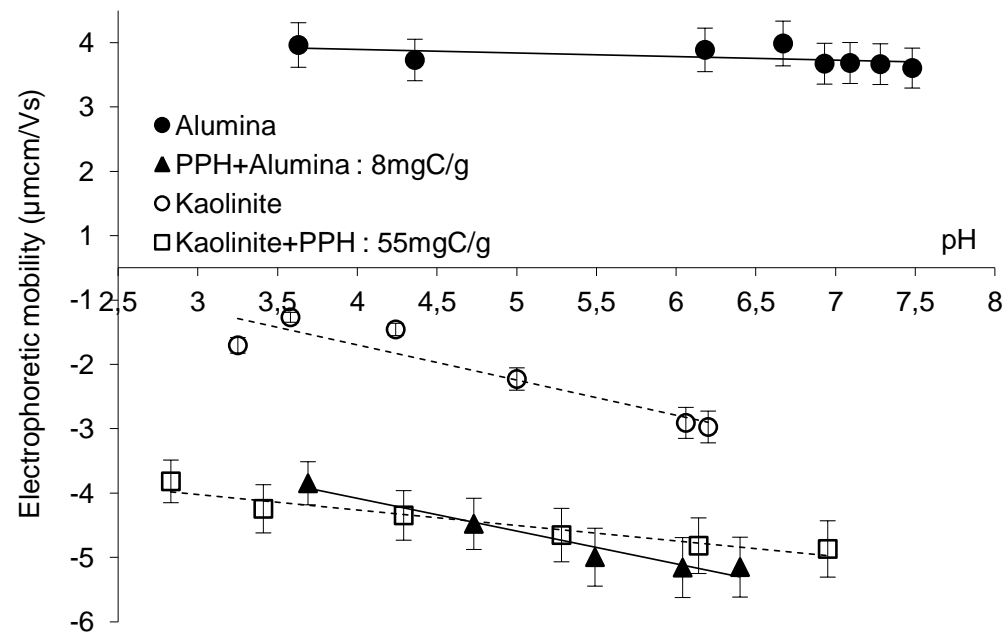

324 Fig. 2: Electrophoretic mobility (EM) of alumina or kaolinite particles as a function of final $\mathrm{pH}$ of mineral325 solution suspensions, in the absence of PPH or at different PPH-to-mineral ratios.

\subsection{ESI(-)-FTMS spectra of initial solution of PPH and chemical formula assignments}

The full mass spectrum recorded in the mass range $120-2000 \mathrm{~m} / \mathrm{z}$ for the native solution of $\mathrm{PPH}$, using the negative ionization mode, showed a mono-modal distribution of peaks lying between $120-800 \mathrm{~m} / \mathrm{z}$ (cf. Fig. A2 in the Appendix). The presence on our mass spectra of peaks at $\mathrm{m} / \mathrm{z}<800$ only is globally consistent with previously published ESI-MS spectra of fulvic acids, which exhibit compounds of molecular weight no higher than a few kDa (Piccolo, 2001; Sutton and Sposito, 2005; Reemtsma, 2009). Several authors have underlined that their mass spectrometry data support the view of HS as being a supramolecular assembly, which can be dissociated during ionization in the ESI probe, in particular when high voltages are applied to the transfer optics (Piccolo et al., 2010). Fig. 3 shows the ESI(-) mass spectra recorded for the native solution of PPH in the ranges $120-400 \mathrm{~m} / \mathrm{z}$ and 400-798 m/z. Circa 1725 and 1190 peaks were detected with $\mathrm{S} / \mathrm{N}>4$ in the aforementioned mass ranges, respectively. The spectra display peaks of high intensity at odd-integral values of $\mathrm{m} / \mathrm{z}$ and peaks of lower intensity at even-integral values of $\mathrm{m} / \mathrm{z}$, with the latter peaks having been reported in previously published studies to correspond to either the ${ }^{13} \mathrm{C}$-containing isotopes of the odd $\mathrm{m} / \mathrm{z}$ ions or to components containing one nitrogen atom (Brown and Rice, 2000; Leenheer et al., 2001; Stenson et al., 2002;

342 Kim et al., 2003). No spacing equal to $(1.0034 / \mathrm{n}) \mathrm{m} / \mathrm{z}$ (with $\mathrm{n}=2,3 \ldots$ ) was detected between the mono343 isotopic peak and the corresponding peak for the one ${ }^{13} \mathrm{C}$-containing isotope, showing that no multiply charged species were generated in the ESI probe during analysis of PPH. The PPH mass spectra display a 
common $14.0156 \mathrm{amu}$ repeating unit due to the existence of homologous series of species differing by $-\mathrm{CH}_{2}$

346 groups (Stenson et al., 2003; Reemtsma et al., 2008; Hertkorn et al., 2008), as reported previously for spectra

347 of DOM analyzed by using FT-ICR MS (Stenson et al., 2002; Stenson et al., 2003; Reemtsma et al., 2008;

348 Hertkorn et al., 2008). Homologous $-\mathrm{CH}_{2}$ series identified for PPH by Kendrick mass analysis were found to 349 contain up to 14 molecules, which suggests that the $-\mathrm{CH}_{2}$ group is an important constitutive element in the structure of FAs (e.g. Fig. A3 in the Appendix). Numerous homologous series were observed and accounted for the large number of different basic structures in $\mathrm{PPH}$, similar to what was reported for DOM samples (Kujawinski, Hatcher, et al., 2002).

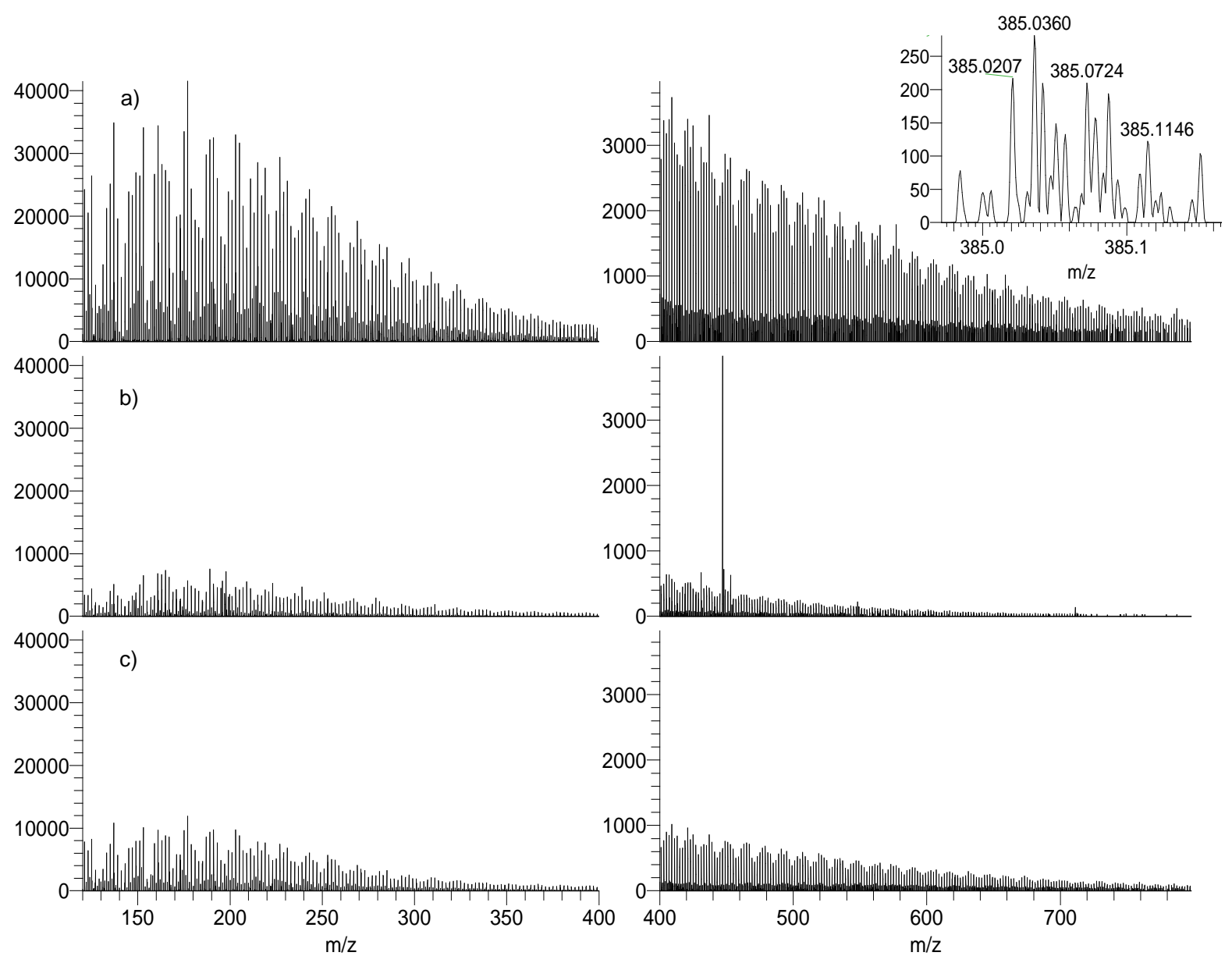

Fig. 3: Negative ion ESI mass spectra in the ranges $120-400 \mathrm{~m} / \mathrm{z}$ and $400-798 \mathrm{~m} / \mathrm{z}$ of PPH native solution (a), and of the supernatants of PPH sorption experiments $(\mathrm{pH}=3.8 \pm 0.1)$ onto alumina $(\mathrm{b})$, and kaolinite $(\mathrm{c})$.

Chemical formulae were assigned to $74 \%$ and $98 \%$ of the PPH compounds detected with $\mathrm{S} / \mathrm{N}>4$ in the mass ranges $120-400 \mathrm{~m} / \mathrm{z}$ and $400-798 \mathrm{~m} / \mathrm{z}$, respectively. Fig. 4 shows that the chemical compositions of PPH compounds are distributed in a wide region of the VK diagrams and belong to the three following categories, 
i.e., polycyclic aromatic compounds (PACs), not-condensed aromatics (NCAs), and compounds of aliphatic character. Such VK diagrams with a wide distribution of compounds is somehow contrasting with most of those previously published for soil humic substances (Rice and MacCarthy, 1991; D’Andrilli et al., 2015) showing data-points gathered in more restricted areas of O/C. However, there are observed in Fig. 4b and Fig. 4a two sets of points that show slightly stronger densities and are mainly made up of strongly oxygenated
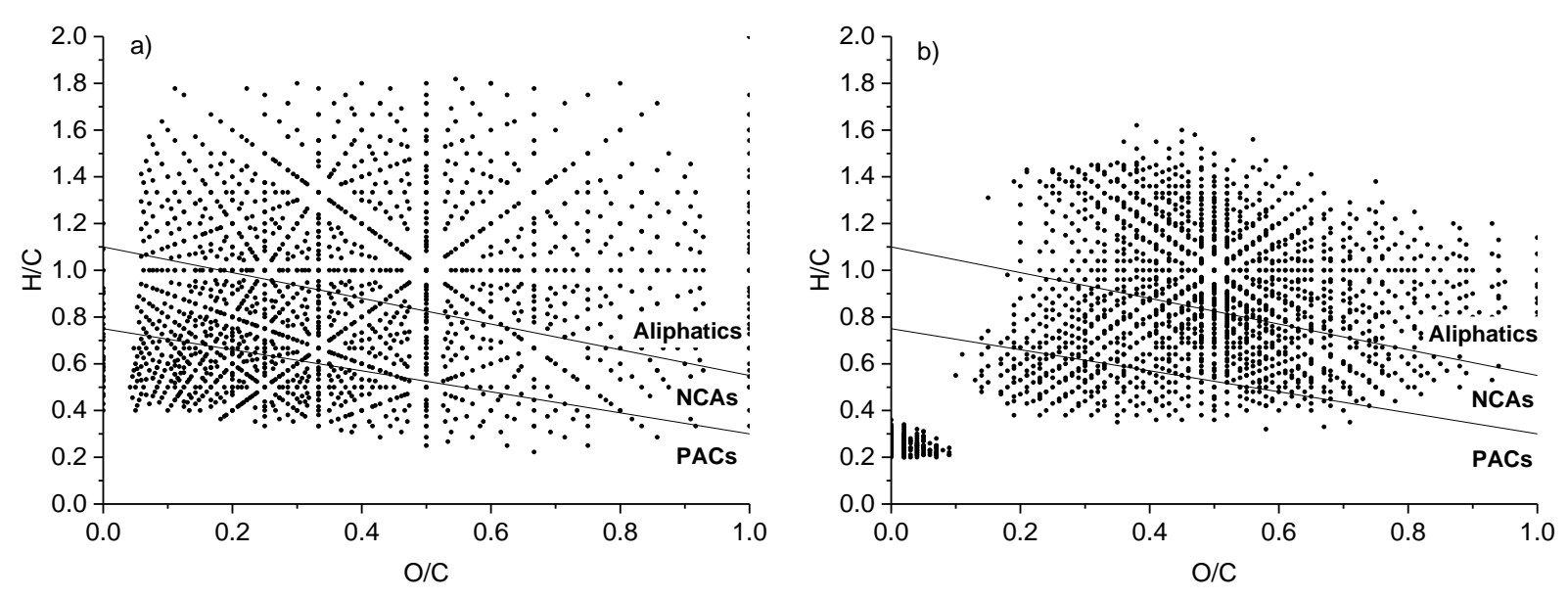

Fig. 4: VK diagram showing elemental formulae assigned to ESI(-) FTMS peaks detected at $\mathrm{pH}=3.8$ on the ranges $120-400 \mathrm{~m} / \mathrm{z}$ (a) and $400-798 \mathrm{~m} / \mathrm{z}$ (b) for the $\mathrm{PPH}$ native solution.

\subsection{Molecular-scale study of the sorptive fractionation of PPH}

\subsubsection{Influence of mineral surface characteristics}

At first examination, ESI(-) spectra provide strong evidence that PPH fractionated during the sorption process.

Fig. 3b and Fig. 3c report spectra of supernatants collected in PPH sorption experiments which were conducted at $\mathrm{pH} 3.8 \pm 0.1$ (and PPH-to-solid ratio of $5 \mathrm{mgC} / \mathrm{g}$ ) and which led to $63 \%$ and $40 \%$ sorption of organic carbon onto $\alpha$-alumina and kaolinite, respectively (Fig. 1). The total ion currents recorded for the mass spectra of the supernatants were lower than those recorded for the initial solution. Moreover, peak intensities were lower in the mass spectra of the supernatants than in those of PPH. Circa $19 \%$ and $11 \%$ of compounds detected with S/N > 4 on the PPH mass spectra were not detected on the supernatants' spectra, 
indicating that the compounds were totally sorbed on the surfaces of alumina and kaolinite during the experiments, respectively. On the contrary, some ions were detected on the mass spectra of the supernatants only. This phenomenon has already been reported by Reiller et al. (2006) and Galindo and Del Nero (2015) in their ESI MS studies of sorption of HA onto hematite and HSs onto alumina, respectively. Actually, the intensity of an ion on a mass spectrum not only depends on its concentration but also on its ionization efficiency and on the matrix, i.e. on the number, the quantity and the structure of the other ions present in the sample. A possible explanation for the detection of some ions in the supernatants' spectra only is that ions undetectable in the native solutions of PPH because of matrix complexity might be ionized more efficiently in the conditions of the supernatants' matrixes due to combined effects of their low sorption onto minerals and of a release of the ionization charge upon preferential sorption of certain PPH compounds. In summary, important features of the spectra were that the extent of the lowering of peak intensities from initial solution to supernatants was varying considerably with the considered ion -going from a peak disappearance to a lowering higher than that of the TIC-, and with the type of mineral. Hence, the degree of sorption of a PPH compound was dependent on both the type of compound and the characteristics of mineral surface.

VK diagrams in Fig. 5 provide information on the order of relative affinity of PPH molecules (sorted as a function of their values of I) for the surfaces of alumina and kaolinite. A main result is that PPH is strongly fractionated between mineral surfaces and solution. Some compounds of low values of $\mathrm{I}(0<\mathrm{I}<0.7$ in Figs. $5 \mathrm{a}, \mathrm{c}$ and $0<\mathrm{I}<0.9$ in Fig. 5b,d) are well sorbed, while those of highest values of $\mathrm{I}(\mathrm{I}>1$ in Figs. $5 \mathrm{a}, \mathrm{c}$ and $\mathrm{I}>1.2$ in Fig. 5b,d) are unambiguously not sorbed at all. Compounds of intermediate values of I are only poorly sorbed or even not sorbed at all (cf. section 2.3.4. for detailed explanation). The fact that distinct ions show very different degrees of sorption on alumina is a first indication that the supramolecular FA structure is not preserved during sorption on this mineral surface, i.e. that the sorption involves relatively strong forces that are likely to modified to some extent the assembly of building blocks holding together thanks to weak cohesion forces. Another main result is that the sorption scheme of PPH onto alumina and kaolinite appear to be quite different.

410 Clear overall trends are observed for alumina (Figs. 5a,b). Firstly, the affinity of a PPH compound for the 411 surface decreases with an increase of its H/C ratio. Secondly, PACs are totally sorbed (I=0) or have a high 412 affinity for the surface $(\mathrm{I}<0.5)$ whereas NCAs and aliphatic compounds exhibit a wide range of I, with the 
413 latter compounds showing the largest distribution. For NCAs, the cloud of data-points is moved towards lower

414 values of $\mathrm{O} / \mathrm{C}$ with increasing values of $\mathrm{I}$, which suggests that increasing amounts of oxygenated

415 functionalities for these molecules increases their affinity for the surface. For the aliphatic compounds, the

416 cloud of data-points is displaced towards both lower values of $\mathrm{O} / \mathrm{C}$ and higher values of $\mathrm{H} / \mathrm{C}$, which suggests

417 that the amount of oxygenated functionalities and/or the length (or number) of aliphatic chains are parameters

418 influencing the affinity for surface. Finally, all the strongly oxygenated molecules $(\mathrm{O} / \mathrm{C}>0.6)$ amongst NCAs,

419 and the H-depleted molecules (H/C $<1.2)$ amongst aliphatics, show a high affinity for the surface of alumina.

420 Differences existing between the sorptive fractionation patterns of PPH in the alumina suspension and

421 kaolinite suspension (Fig. 5c,d) are rather striking. Firstly, the totally sorbed compounds $(\mathrm{I}=0)$ are fewer for

422 kaolinite than for alumina and they are moreover dispersed over a wide region of VK diagram. Secondly, no 423 effect of the $\mathrm{H} / \mathrm{C}$ ratio on the sorption is observed. Unlike for alumina, compounds showing an affinity for the 424 surface of kaolinite extend to aliphatic compounds of intermediate $\mathrm{O} / \mathrm{C}$ and high $\mathrm{H} / \mathrm{C}$-and include as well the 425 same compounds as those displaying a high affinity for alumina, such as NCAs with O/C $>0.4$ and aliphatics 426 with $\mathrm{O} / \mathrm{C}>0.6$ (together with some PACs of $0.2<\mathrm{O} / \mathrm{C}<0.6$ )-. It is also to be noted that most of the 427 compounds sorbed onto kaolinite show I values in a narrow range $(0.5<\mathrm{I}<0.9)$. All these features clearly 428 indicate that the surface of kaolinite is weakly selective, and that unlike for alumina, the supramolecular 429 structure of the FA is likely to be globally preserved during sorption onto kaolinite. Thirdly, another striking 430 difference between kaolinite and alumina is that a great part of PACs, including those having weak $\mathrm{O} / \mathrm{C}$ ratios, 431 are only slightly sorbed or not sorbed at all onto kaolinite (Fig. $5 c, 0.7<\mathrm{I}<1$ ). All the differences described 432 above suggest that different molecular characteristics and sorption mechanisms are influencing the sorptive 433 fractionation of PPH onto kaolinite and alumina. 

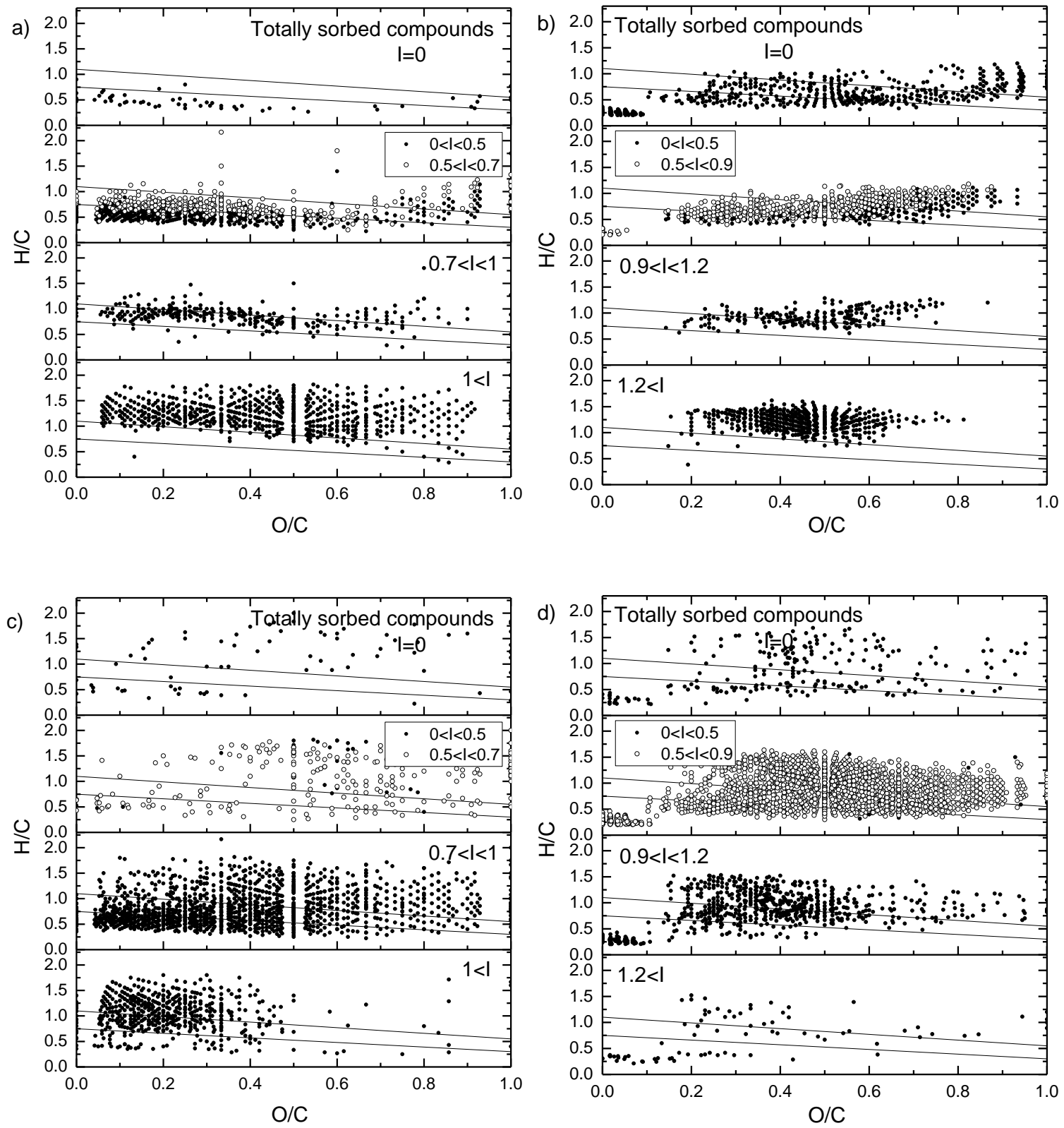

437 Fig. 5: VK diagrams for PPH compounds sorted as a function of their relative affinity for the surface of alumina at pH $3.9(\mathrm{a}, \mathrm{b})$ and for the surface of kaolinite at pH $3.7(\mathrm{c}, \mathrm{d})$ for the ranges $120-400 \mathrm{~m} / \mathrm{z}$ and 400 $798 \mathrm{~m} / \mathrm{z}$, respectively.

\subsubsection{Relative affinity order for mineral surfaces of $\mathrm{PPH}$ compounds in $-\mathrm{CO}_{2}$ and $-\mathrm{CH}_{2}$ series}

443 Relations existing between the sorption degree of a molecule in a $-\mathrm{CO}_{2}$ series and its number of $\mathrm{CO}_{2}$ groups, 444 as representative for the number of carboxyl functionalities, or between the sorption degree of a molecule in $445 \mathrm{CH}_{2}$ series and its number of $-\mathrm{CH}_{2}$ groups, as representative for length/number of aliphatic chains, were 446 investigated to gain further information on the mechanisms involved in the sorption of PPH onto alumina and 
447 kaolinite. $-\mathrm{CO}_{2}$ series or $-\mathrm{CH}_{2}$ series are made up by compounds whose chemical formula differ only by their

448 number of $\mathrm{CO}_{2}$ or $\mathrm{CH}_{2}$, respectively.

450 Figs. 6a,b report, for alumina-solution systems at $\mathrm{pH} 3.9$, the evolution of $\mathrm{I}$ as a function of number of carbon 451 atoms $\left(\mathrm{n}_{\mathrm{C}}\right)$ of PPH compounds in $-\mathrm{CO}_{2}$ series of aliphatic compounds or oxygenated NCAs compounds $452(\mathrm{O} / \mathrm{C}>0.2)$, and in $-\mathrm{CO}_{2}$ series of PACs or poorly-oxygenated NCAs, respectively. Figs. $6 \mathrm{c}, \mathrm{d}$ refer to the 453 results obtained for the $-\mathrm{CH}_{2}$ series. A striking result is the difference in the $\mathrm{n}_{\mathrm{C}}-\mathrm{I}$ relations existing between the 454 two sets of series. For the aliphatic compounds or the NCAs with $\mathrm{O} / \mathrm{C}>0.2$ (Fig.6a), the $-\mathrm{CO}_{2}$ series show an 455 inverse correlation between the value of I and the number of carbon atoms, $\mathrm{n}_{\mathrm{C}}$. This indicates that the degree 456 of sorption of a molecule in the series is directly related to its number of carboxyl groups. For the $-\mathrm{CH}_{2}$ series, 457 a sharp increase of $I$ with $n_{C}$ was observable (Fig. 6c) indicating that the affinity for surface of a molecule 458 within a series decreases with an increasing of the length/number of aliphatic chains. Such remarkable and monotonous $\mathrm{n}_{\mathrm{C}}-\mathrm{I}$ relations, which were observed for all the $-\mathrm{CO}_{2} /-\mathrm{CH}_{2}$ series investigated, suggest that a single mechanism controls the degree of sorption onto alumina of aliphatic compounds and oxygenated NCAs. The fact that distinct molecules of a same $-\mathrm{CO}_{2} /-\mathrm{CH}_{2}$ series display very different degrees of sorption -some being totally sorbed $(\mathrm{I}=0)$ and others being not sorbed at all $(\mathrm{I}>1.2)$ - clearly indicate that the supramolecular structure of the FA existing in solution is greatly modified during sorption. Probably, the sorption implies strong forces which compete efficiently against the weak cohesion forces of the supramolecular assemblies, leading to different sorption behaviors for the distinct FA building blocks at the surface of alumina. Regarding the PACs and the poorly-oxygenated NCAs, more complex features were observed. The degree of sorption of a molecule within a $-\mathrm{CH}_{2}$ series was found to be only weakly correlated with its number of $\mathrm{CH}_{2}$ groups (only a small increase of $\mathrm{I}$ with $\mathrm{n}_{\mathrm{c}}$ is observable in Fig. $6 \mathrm{~d}$ ). In contrast, $-\mathrm{CO}_{2}$ series showed a noticeable and not monotonous evolution of $\mathrm{I}$ with $\mathrm{n}_{\mathrm{C}}$. Indeed, bell-shaped curves were observed: the last members of the $-\mathrm{CO}_{2}$ series showed a decrease of I with an increase of $\mathrm{n}_{\mathrm{C}}$ while the first members of the series showed the opposite (Fig. 6b). Hence, PACs and NCA compounds with O/C $<0.2$ show different sorption trends according to their molecular $\mathrm{O} / \mathrm{C}$ ratio, which suggests that within a $-\mathrm{CO}_{2}$ series of compounds two different mechanisms of sorption (at least) operate, one favoring the retention of molecules containing some oxygen and another favoring the retention of molecules depleted in oxygen. 

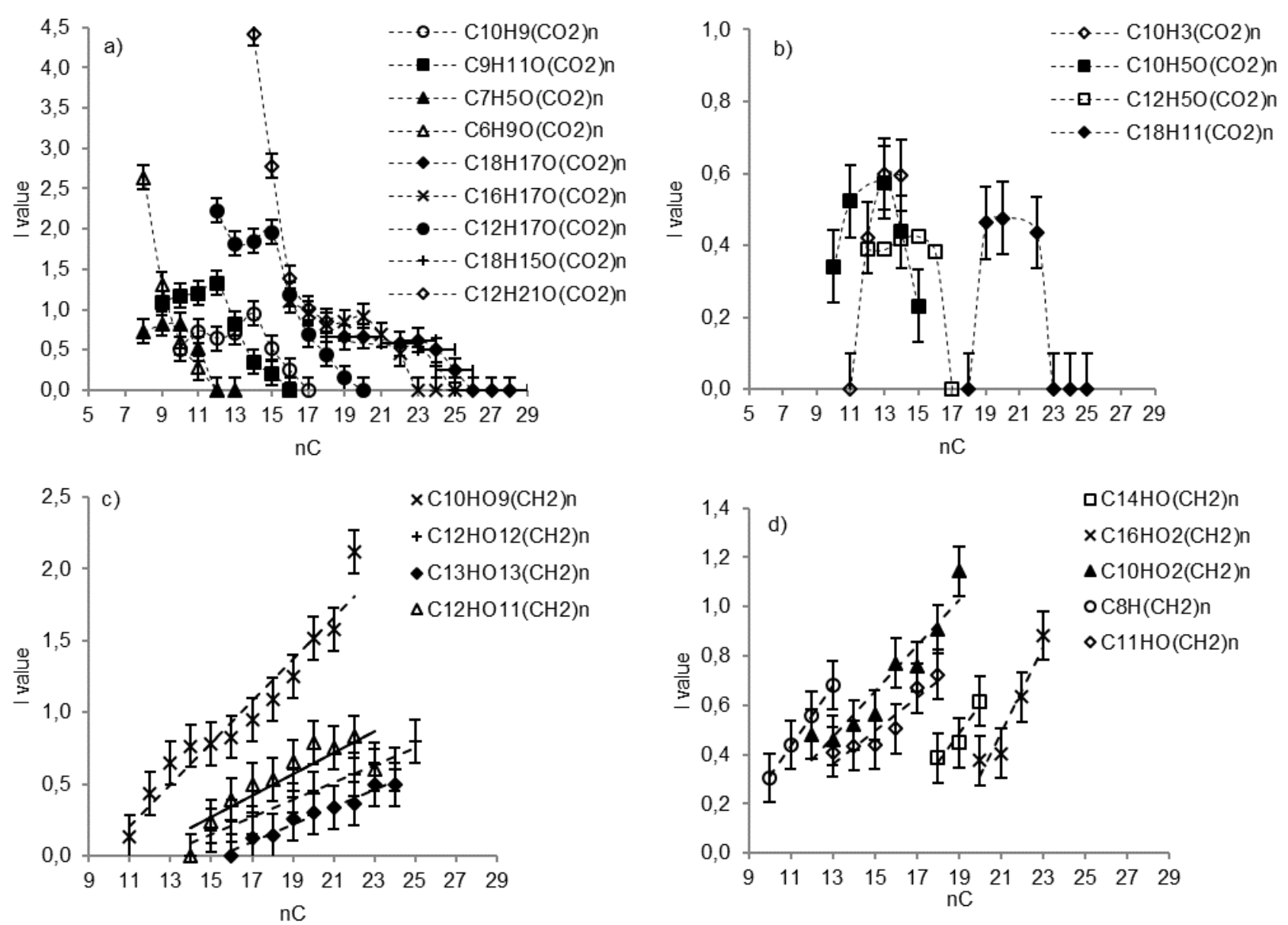

Fig. 6: Evolution of value of I of PPH compounds as a function of number of carbon atoms $\left(\mathrm{n}_{\mathrm{C}}\right)$ for aliphatics and NCAs with $\mathrm{O} / \mathrm{C}>0.2$ in $-\mathrm{CO}_{2}$ (a) and $-\mathrm{CH}_{2}$ (c) series, and for NCAs depleted in oxygen and PACs in $\mathrm{CO}_{2}$ (b) and $-\mathrm{CH}_{2}$ (d) series, in alumina-solution systems at $\mathrm{pH}$ 3.9.

Figs. 7a,b show, for kaolinite-solution systems at $\mathrm{pH} 3.7$, plots of $\mathrm{I}$ versus $\mathrm{n}_{\mathrm{c}}$ for $-\mathrm{CO}_{2}$ series of aliphatic compounds or oxygenated NCAs compounds $(\mathrm{O} / \mathrm{C}>0.2)$ and for $-\mathrm{CO}_{2}$ series of PACs or poorly-oxygenated NCAs, respectively. The same series as those described above for alumina were considered for kaolinite. The most striking result is that the values of I for $-\mathrm{CO}_{2}$ series of aliphatics or highly oxygenated NCAs (Fig. 7a) are in a very limited range, showing that all compounds in a series have almost a similar relative affinity for the surface of kaolinite. Such a trend in kaolinite-solution systems is also visible for the $-\mathrm{CO}_{2}$ series of PACs and oxygen-depleted NCA molecules (Fig. 7b). These results contrast markedly with those reported above for the surface of alumina, for which values of I varied largely amongst compounds of a $-\mathrm{CO}_{2}$ series (cf. Figs. 6a,b). It is also to be noted that, in the PPH-kaolinite system, none of the $-\mathrm{CO}_{2}$ series investigated showed well-sorbed molecules with values of I lower than 0.5. All these results confirm that the clay surface is less selective than the Al-oxide surface and that molecular characteristics such as number of $-\mathrm{CO}_{2}$ groups and/or 
number/length of aliphatic chains are not determining parameters regulating the sorptive fractionation of PPH onto kaolinite.

494
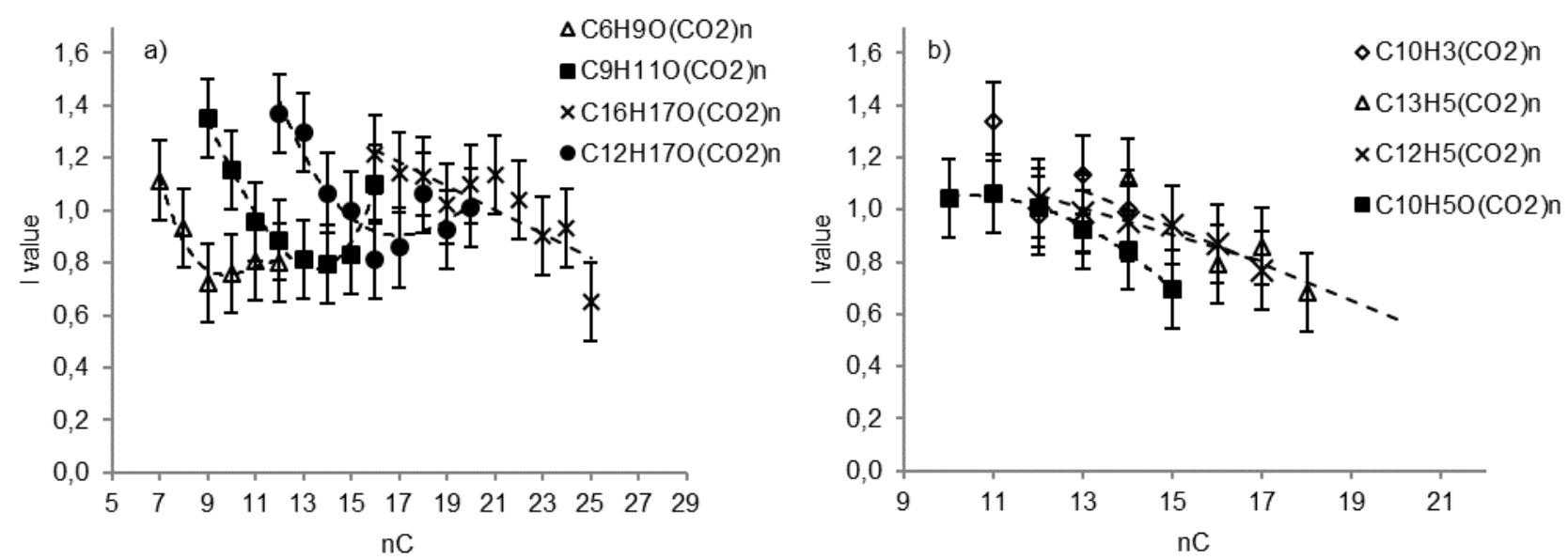

Fig. 7: Evolution of the values of I within $-\mathrm{CO}_{2}$ series of aliphatics or NCAs with $\mathrm{O} / \mathrm{C}>0.2$ (a), and $-\mathrm{CO}_{2}$

series of NCAs depleted in oxygen and PACs (b), after sorption of PPH onto kaolinite at pH 3.7.

\subsubsection{Effect of $\mathrm{pH}$ on the sorptive fractionation of $\mathrm{PPH}$}

Regarding the sorption onto alumina, it appears that the relative affinities of PPH molecules for the surface do not change significantly in the acidic $\mathrm{pH}$ range $3-5$, as shown by comparing VK diagrams obtained for the system at $\mathrm{pH}=4.8$ (Figs. 8a,b) and at $\mathrm{pH}=3.9$ (cf. Figs. 5a,b). Correlations observed at $\mathrm{pH} 4.8$ between the degree of molecule sorption and molecular parameters within $-\mathrm{CO}_{2}$ or $-\mathrm{CH}_{2}$ series (data not shown) were similar to those described for the $\mathrm{pH} 3.9$ (cf. paragraph 3.3.2).

Regarding the sorption onto kaolinite, it is first to be noted that fewer compounds are represented on the VK diagrams reporting the relative affinities of PPH molecules for surface at pH 5.0 (Figs. 8c,d) than at pH 3.7 (Figs. 5c,d). This is due to the fact that supernatants for the kaolinite-PPH-solution systems had to be analyzed at $\mathrm{pH} 5.0$ (cf. section 2.2), which leads to an alteration of the MS response (for illustrative purposes, compare VK diagrams of native PPH solution analyzed at pH 3.8 in Fig. 4 and at pH 5.0 in Fig. A4 in the Appendix). Despite fewer compounds are detected for the native solution of PPH at $\mathrm{pH} 5.0$ than at $\mathrm{pH} 3.8$, some key trends in the pH-dependency of the sorption of PPH onto kaolinite can still be determined. Some highly oxygenated aliphatics and NCAs are poorly sorbed at $\mathrm{pH} 5.0$ (while they display a good affinity for the 
surface at $\mathrm{pH}$ 3.7). This suggests the existence of a process that competes against sorption at $\mathrm{pH} 5.0$ and tends

516 to keep these highly oxygenated molecules in solution. Interestingly, the final concentration of dissolved

517 aluminum in kaolinite-PPH suspensions at $\mathrm{pH} 5.0$ was found to be ca. four times higher than in kaolinite

518 suspensions without PPH (cf. Fig. A5 in the Appendix). This supports the formation in kaolinite-PPH systems

519 of solution complexes implying the most oxygenated aliphatic and NCA compounds and the dissolved 520 aluminum originating from the dissolution of kaolinite.
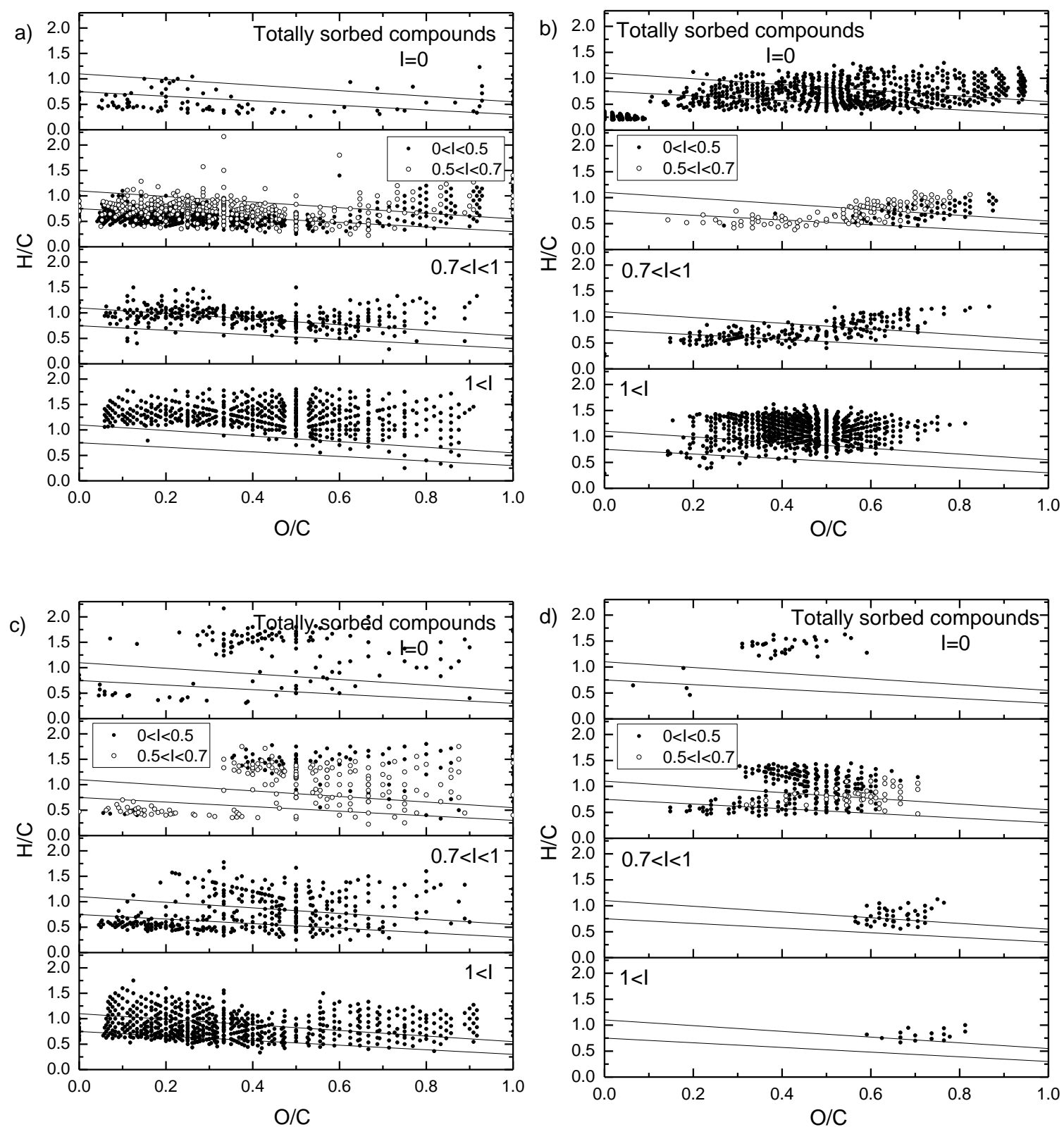

524 Fig. 8: VK diagrams for PPH compounds sorted as a function of their relative affinity for the surface of 525 alumina at $\mathrm{pH} 4.8(\mathrm{a}, \mathrm{b})$ and for the surface of kaolinite at $\mathrm{pH} 5.0(\mathrm{c}, \mathrm{d})$ for the ranges $120-400 \mathrm{~m} / \mathrm{z}$ and 400 $526798 \mathrm{~m} / \mathrm{z}$, respectively. 


\section{DISCUSSION}

530 A main finding is that the trends in the sorptive fractionation of PPH in kaolinite-solution systems are very 531 different than those observed for alumina, in the present work or in previously published studies of molecularscale alumina-HS-interactions (Galindo and Del Nero, 2014; Galindo and Del Nero, 2015). Such a finding provides strong evidence that the mineral surface properties are a key parameter governing the sorptive fractionation of HS.

535 All the results obtained at the molecular scale for the kaolinite-solution system suggest that the sorption of 536 PPH onto kaolinite is mostly the result of a weakly selective mechanism. For example, the dispersion of the 537 totally sorbed PPH compounds over a wide region of VK diagram and the closeness of the I values of the 538 molecules in $-\mathrm{CO}_{2}$ series well exemplify that the number of $\mathrm{CO}_{2}$ groups in a molecule and, more generally, molecular acidity, have a slight influence on the degree of sorption of NCAs and aliphatics onto kaolinite.

540 These features rule out a substantial contribution to PPH sorption of a surface ligand exchange mechanism 541 between the hydroxyl groups existing on the kaolinite surface and the carboxyl groups of PPH. Though, it has 542 been widely reported that high affinity aluminol sites on singly coordinated oxygens at edges and steps of 543 kaolinite surface have chemical properties close to the surface sites of alumina (e.g. Kummert and Stumm, 544 1980). These sites probably have a strong affinity for the highly oxygenated compounds of PPH but are not 545 numerous enough to have a measurable effect on the overall sorptive fractionation pattern of PPH.

546 Based upon the structure of fulvic acid molecules, which have numerous functional groups with hydrogens 547 bound to heteroatoms $(\mathrm{N}, \mathrm{O})$, hydrogen bonding seems to be a good candidate because such a mechanism is 548 not as selective as surface ligand exchange. Hydrogen bonding well accounts for the lack of preferential 549 sorption observed amongst all aliphatics and NCA molecules of O/C > 0.2 (Fig. 5d). Sorption of most of PPH 550 compounds, including the hydrogenated compounds, may thus imply aluminol or silanol groups existing on 551 the doubly coordinated oxygens located on the basal planes of kaolinite, which are poorly selective sites expected to be involved in weak sorption mechanisms such as hydrogen bonding. Probably, such weak 553 sorption mechanisms do not compete efficiently against the cohesion forces of the supramolecular FA 554 assemblies. The supramolecular structure would thus be globally preserved during sorption, accounting for the 555 similar affinities of the different FA compounds for the surface of kaolinite. Implication of these low affinity 
sites not only accounts for a weak fractionation amongst members of $-\mathrm{CO}_{2}$ series of aliphatics or NCAs during their sorption onto kaolinite, but also for the very low degree of sorption of PACs. No positive $\mathrm{n}_{\mathrm{c}}-\mathrm{I}$ correlation were observed in kaolinite-solution systems for $-\mathrm{CO}_{2}$ series of PACs with low $\mathrm{O} / \mathrm{C}$ ratios, suggesting a poor contribution of hydrophobic interactions between PACs and kaolinite surface. Possibly, the rather limited degree of sorption onto kaolinite of the PACs bearing oxygenated functionalities (due to low amounts of high affinity surface aluminol sites) resulted in few amounts of hydrophobic domains available, in the first layer of PPH molecules sorbed at the clay surface, for further interactions with hydrophobic moieties of NCAs and PACs of low $\mathrm{O} / \mathrm{C}$ present in solution. Moreover, the first layer of sorbed compounds is only weakly bonded on the basal planes of the kaolinite surface, preventing a strong attach of poorly-oxygenated PACs on the surface.

Implication of the low affinity sites on the basal planes of kaolinite also accounts for the pH-dependency of the sorption of the highly oxygenated aliphatic and NCA compounds of PPH. A decrease of the degree of sorption of these molecules at $\mathrm{pH} 5.0$ results from their complexation with aluminum in solution competing against their sorption at the surface of the mineral. Indeed, an increase in $\mathrm{pH}$ value induces the deprotonation of both the aluminol/silanol groups on the basal planes of kaolinite, as well as of the carboxyl groups of PPH.

571 Such a deprotonation has the effect of preventing hydrogen bonding of PPH molecules on the kaolinite 572 surface sites, and of decreasing the degree of sorption of the highly oxygenated aliphatic and NCA 573 compounds. Moreover, the deprotonation of the carboxyl groups of these molecules also favors the formation 574 of FA-aluminum complexes in solution. The pH-dependency of the sorption of highly oxygenated aliphatic and NCA compounds in PPH is thus the result of both these processes.

576 The molecular-scale findings on the sorptive fractionation trends of PPH during sorption at the clay surface 577 are quite contrasting with those observed for the alumina-solution-PPH systems, for which molecules showing 578 the highest affinity for the mineral surface are the PACs and the highly oxygenated compounds amongst 579 aliphatics or NCAs. For the latter compounds, strong evidence is provided that a single mechanism controls 580 their sorption at the alumina surface, as shown, for example, by the remarkable positive correlations existing for PPH compounds in $\mathrm{CO}_{2}$ series between the degree of sorption of a molecule and its molecular $\mathrm{O} / \mathrm{C}$ ratio.

582 Highly-oxygenated NCA and aliphatic compounds carry multiple carboxyl and phenol groups which are well 583 known to be involved in ligand exchange reactions (specific adsorption) with surface aluminol groups, e.g. 584 (Schlautman and Morgan, 1994). The positive correlation observed between affinity for the surface of alumina 
and number of carboxyl groups of a PPH compound in a $-\mathrm{CO}_{2}$ series even includes molecules containing up to

5865 groups or more (e.g. Fig. 6). As discussed in previously published papers by our team (Galindo and Del

587 Nero, 2014), it is unlikely that more than three of carboxyl groups of a molecule are involved simultaneously

588 in multiple bonding with aluminol groups at the surface of alumina. The concomitant increase of affinity for

589 alumina surface and $\mathrm{nCO}_{2}$ of compounds in $\mathrm{CO}_{2}$-series of the types highly-oxygenated NCAs or aliphatics is

590 thus due likely to a change in molecule acidity. Such an interpretation was already proposed for simple

591 organic acids by Evanko and Dzombak (1998), who suggested that enhanced sorption with increasing number

592 of carboxyl groups of acids was due to changes in the acidity of compound rather than to multiple bonding,

593 and was supported by the results of Janot et al. (2012). The interpretation is also supported by our results

594 showing that a lengthening of aliphatic chains of a molecule within a $-\mathrm{CH}_{2}$ series, which is expected to

595 decrease the molecule acidity due to donor inductive effect, decreases the affinity for the surface of alumina.

596 Regarding the NCAs with $\mathrm{O} / \mathrm{C}<0.2$ and the PACs, the bell shape of the curves reporting affinity of

597 molecules in $\mathrm{CO}_{2}$-series for alumina surface vs. number of carboxyl groups indicate that different mechanisms

598 are responsible for the sorption of molecules of lowest $\mathrm{O} / \mathrm{C}$ ratios and highest $\mathrm{O} / \mathrm{C}$ ratios, respectively. For the

599 latter molecules, acidity is the key parameter controlling the degree of sorption (same types of $\mathrm{n}_{\mathrm{C}} \mathrm{I}$ inverse

600 correlation as those observed for the aliphatics), and surface ligand exchange is the mechanism driving the

601 sorption. For the former compounds, the positive $\mathrm{n}_{\mathrm{c}}$-I correlation observed rules out acidity as a parameter

602 governing sorption. The existence of this correlation for compounds having many hydrophobic moieties (e.g.

603 aromatic structures) and few oxygenated functional groups provides strong evidence that their degree of

604 hydrophobicity determines their affinity for the surface of the oxide. A possible explanation is as follows. As

605 evidenced by the impact of molecule acidity on the degree of sorption of a FA molecule onto alumina, - either

606 for aliphatics, NCAs and PACs-, the supramolecular structure of FA appears to be not preserved during

607 sorption, allowing : (i) the sorption of the most highly acidic FA compounds to be sorbed via surface ligand

608 exchange involving their hydrophilic moieties and (ii) the release into solution of PACs with very low O/C

609 and their subsequent sorption via interaction between their hydrophobic moieties and those of the strongly

610 sorbed PACs (which form a first sorption layer onto alumina). Another possible interpretation is that the

611 electric fields induced by the surface charge of alumina influence the dielectric constant of water (Booth,

612 1951). Water at the surface of alumina would thus have a lower apparent dielectric constant, which favors the

613 adsorption of hydrophobic molecules. Molecular-scale evidence that the degree of hydrophobicity governs the 
614 degree of sorption of poorly-oxygenated PACs and NCAs onto a metallic oxide has been published recently

615 for a peat humic acid (Galindo and Del Nero, 2015) but has never been so far demonstrated for fulvic acids.

616 The molecular-scale results presented here for the sorption of PPH are useful for rationalizing the contrasting

617 results of spectroscopic studies published on the sorption of bulk FAs at the surfaces of metallic (hydr)oxides

618 and clays, respectively. Ghosh et al. (2009) studied by spectroscopy techniques the fractionation behavior of

619 HS on minerals with different surface properties. They reported that aliphatic fractions of HS are more prone

620 to sorption at the surface of clay minerals than the highly aromatic fractions, while carboxylic functional

621 groups play a significant role in the sorption of HA on goethite. Spectroscopic studies have long reported

622 preferential sorption of aromatic structures bearing oxygenated functionalities of HS at the surface of metallic

623 (hydr)oxides (Meier et al., 1999; Zhou et al., 2001; Kaiser, 2003; Claret et al., 2008). For example, Kaiser

624 showed for HS-solution-goethite systems a preferential removal from solution of aromatic and carboxyl C,

625 whereas alkyl-C accumulated in the solution. Claret et al. (2008) reported a preferential sorption at the surface

626 of alumina of the hydrophobic fractions of FA and/or of the FA compounds showing high contents in aromatic

627 moieties and functional groups, as compared to aliphatic fractions. In the present study, on the one hand, we

628 provide molecular-scale evidence that most of the acidic, aliphatic compounds of a FA display a greater

629 affinity for the kaolinite surface than PACs because the most effective mechanism of sorption of FA

630 molecules at such a clay surface is a weak sorption via H-bonds at the aluminol / silanol sites on the doubly

631 coordinated oxygens located on the basal planes of the clay. On the other hand, the present molecular-scale

632 study also confirms unequivocally that clear relationships exist between molecular parameters such as acidity

633 or hydrophobicity of a molecule and its degree of sorption in a metallic oxide-solution system. The sorptive

634 fractionation of FAs in such systems, in contrast to clay systems, is due to a competition between protons and

635 metallic centers at the surface of the mineral for coordination of FA molecules, and to the subsequent sorption

636 of poorly-oxygenated PACs by hydrophobic forces involving hydrophobic moieties in the first layer of sorbed

637 FA molecules on the oxide surface.

638

639

\section{CONCLUSION}

641 The results of the present study on the fractionation behavior of a soil FA onto alumina and kaolinite provide 642 valuable molecular-scale insights on the relationships existing between the chemical characteristics of the 
643 molecules and their distribution between aqueous and mineral phases, as well as on the distinct mechanisms

644 involved in the fractionation of a FA during its sorption onto minerals of different surface properties. A main

645 finding is that the molecular-scale fractionation of FA in kaolinite suspensions is highly contrasting with that

646 observed here for alumina or with those reported in previously published studies for aluminum oxide (Galindo

647 and Del Nero, 2014; Galindo and Del Nero, 2015). Such a finding highlights that the key parameter

648 controlling the sorptive fractionation trends of FAs is the nature, i.e. oxides vs. clays, and the surface

649 properties of sorbing minerals.

651 The data presented here confirm previous work by our team (Galindo and Del Nero, 2014) indicating that 652 acidity of FA molecules is a main parameter determining the relative degree of sorption onto Al-oxides of FA 653 molecules, with the highly acidic molecules bearing oxygenated functionalities being sorbed preferentially via 654 surface ligand exchange involving the high-affinity aluminol surface sites. Our study also reveals that degree 655 of hydrophobicity of PACs and NCAs of low $\mathrm{O} / \mathrm{C}$ ratio is another key molecular parameter influencing the 656 degree of sorption of these FA compounds, which are able to interact with hydrophobic domains of organic 657 molecules (probably mainly PACs) strongly retained at the Al oxide surface within a first layer of 658 specifically-sorbed organic molecules. The present study establishes that, whatever the FA composition and in 659 a range of acidic $\mathrm{pH}$ values, surface ligand exchange and hydrophobic interactions are the main (cooperative) 660 mechanisms leading to the preferential retention on metal oxides of most of PACs, of NCAs with high O/C 661 ratios $(>0.5)$ and of highly-oxygenated aliphatic compounds $(\mathrm{O} / \mathrm{C}>0.7)$, whereas hydrogenated and less oxygenated NCAs and aliphatics are left in the solution.

664 The first molecular-scale description presented here of the fractionation of a FA in a clay mineral-solution 665 system reveals that, unlike for metallic oxides, molecular acidity has no substantial effect on the degree of 666 sorption of a FA molecule onto a clay, and that surface ligand exchange is not the mechanism driving the 667 sorptive fractionation of FA in such systems. Instead, the main mechanism of sorption is hydrogen bonding of 668 FA molecules at the low affinity sites (aluminol or silanol) present on the basal planes of the clay, as 669 evidenced here by the weak sorptive fractionation existing amongst members of $-\mathrm{CO}_{2}$ homologous series of 670 aliphatics or NCAs during their sorption onto kaolinite at low pH. In contrast, at slightly acidic pH (5), a 671 fractionation occurs to a certain extent between molecules within a same class of FA compounds (for 
aliphatics as well as NCAs), due to a decreasing with $\mathrm{pH}$ of the degree of sorption of highly acidic molecules

673 bearing oxygenated functionalities. Such a fractionation is explained by the fact that dissolved Al has the 674 ability to compete successfully for the coordination of such reactive organic molecules against a weak 675 sorption process such as H-bonding. Finally, the predominance of a weak and poorly-selective mechanism of 676 sorption of FA molecules like H-bonding indirectly accounts for a very low degree of sorption of PACs or of 677 low $\mathrm{O} / \mathrm{C}$ NCAs onto kaolinite. Absence of preferential and specific sorption at surface of kaolinite of aromatic 678 structures activated by oxygenated functionalities prevents the formation of a first layer of strongly-sorbed 679 molecules exhibiting hydrophobic domains which would be available for further sorption of FA compounds 680 via hydrophobic interactions.

The results of the present molecular scale studies have several implications on our knowledge of storage of natural organic matter in soils as well as on the cycle of metals. It is to be expected that clayey soils trap lesser amounts of condensed aromatics than metal oxide-rich soils and may retain non-selectively most of FA compounds of the types of aliphatics or not-condensed aromatics at acidic $\mathrm{pH}$, via weak sorption mechanisms. In turn, solutions percolating through clayey soils, if being slightly acidic, would contain a multiplicity of the FA organic acids bearing multiple oxygenated functionalities, owing to the high ability of these FA molecules for binding dissolved metals and their only weak sorption at clay surfaces. Such a feature would promote the transport of metal pollutants with soil solutions. Consequently, (long term) metal sorption capacities of clayey soils are expected to be rather low compared to that of soils rich in $\mathrm{Al}$ oxi-hydroxides.

\section{ACKNOWLEDGEMENTS}

693 We thank IPHC and the Alsace Region for their financial support. We gratefully acknowledge the reviewers 694 for their helpful comments, and for improvement of the manuscript.

\section{REFERENCES}

Aiken G. R., McKnight D. M., Wershaw R. L. and MacCarthy P. (1985) Humic substances in soil, sediment, and water: geochemistry, isolation and characterization., Wiley.

Booth F. (1951) The Dielectric Constant of Water and the Saturation Effect. J. Chem. Phys. 19, 391-394. 
Brown T. L. and Rice J. A. (2000) Effect of Experimental Parameters on the ESI FT-ICR Mass Spectrum of Fulvic Acid. Anal. Chem. 72, 384-390.

Bryan N. D., Abrahamsen L., Evans N., Warwick P., Buckau G., Weng L. and Van Riemsdijk W. H. (2012) The effects of humic substances on the transport of radionuclides: Recent improvements in the prediction of behaviour and the understanding of mechanisms. Appl. Geochem. 27, 378-389.

Claret F., Schäfer T., Brevet J. and Reiller P. E. (2008) Fractionation of Suwannee river fulvic acid and aldrich humic acid on a-Al2O3: Spectroscopic evidence. Environ. Sci. Technol. 42, 8809-8815.

D'Andrilli J., Chanton J. P., Glaser P. H. and Cooper W. T. (2010) Characterization of dissolved organic matter in northern peatland soil porewaters by ultra high resolution mass spectrometry. Org. Geochem. 41, 791-799.

D'Andrilli J., Cooper W. T., Foreman C. M. and Marshall A. G. (2015) An ultrahigh-resolution mass spectrometry index to estimate natural organic matter lability: FTICRMS organic matter molecular lability index. Rapid Commun. Mass Spectrom. 29, 2385-2401.

Del Nero M., Galindo C., Barillon R., Halter E. and Madé B. (2010) Surface reactivity of a-Al2O3 and mechanisms of phosphate sorption: In situ ATR-FTIR spectroscopy and $\zeta$ potential studies. J. Colloid Interface Sci. 342, 437-444.

Dobbs J. C., Susetyo W., Knight F. E., Castles M. A., Carreira L. A. and Azarraga L. V. (1989) Characterization of metal binding sites in fulvic acids by lanthanide ion probe spectroscopy. Anal. Chem. 61, 483-488.

Engebretson R. R. and von Wandruszka R. (1994) Micro-organization in dissolved humic acids. Environ. Sci. Technol. 28, 1934-1941.

Evanko C. R. and Dzombak D. A. (1998) Influence of structural features on sorption of NOManalogue organic acids to goethite. Environ. Sci. Technol. 32, 2846-2855.

Fairhurst A. J. and Warwick P. (1998) The influence of humic acid on europium-mineral interactions. Colloids Surf. Physicochem. Eng. Asp. 145, 229-234.

Feng X., Simpson A. J. and Simpson M. J. (2005) Chemical and mineralogical controls on humic acid sorption to clay mineral surfaces. Org. Geochem. 36, 1553-1566.

Filius J. D., Meeussen J. C., Lumsdon D. G., Hiemstra T. and van Riemsdijk W. H. (2003) Modeling the binding of fulvic acid by goethite: the speciation of adsorbed FA molecules. Geochim. Cosmochim. Acta 67, 1463-1474.

Fu H., Quan X., Chen S., Zhao H. and Zhao Y. (2005) Interaction of humic substances and hematite: FTIR study. J. Environ. Sci. 17, 43-47.

Galindo C. and Del Nero M. (2015) Chemical fractionation of a terrestrial humic acid upon sorption on alumina by high resolution mass spectrometry. RSC Adv 5, 73058-73067.

Galindo C. and Del Nero M. (2014) Molecular Level Description of the Sorptive Fractionation of a Fulvic Acid on Aluminum Oxide Using Electrospray Ionization Fourier Transform Mass Spectrometry. Environ. Sci. Technol. 48, 7401-7408.

Ghosh S., Wang Z.-Y., Kang S., Bhowmik P. C. and Xing B. S. (2009) Sorption and fractionation of a peat derived humic acid by kaolinite, montmorillonite, and goethite. Pedosphere 19, 21-30. 
Gu B., Schmitt J., Chen Z., Liang L. and McCarthy J. F. (1995) Adsorption and desorption of natural organic matter on iron oxide: mechanisms and models. Environ. Sci. Technol. 28, 38-46.

Hertkorn N., Frommberger M., Witt M., Koch B. P., Schmitt-Kopplin P. and Perdue E. M. (2008) Natural Organic Matter and the Event Horizon of Mass Spectrometry. Anal. Chem. 80, 8908-8919.

Hur J. and Schlautman M. A. (2004) Effects of $\mathrm{pH}$ and phosphate on the adsorptive fractionation of purified Aldrich humic acid on kaolinite and hematite. J. Colloid Interface Sci. 277, 264-270.

Janot N., Reiller P. E. and Benedetti M. F. (2013) Modelling Eu(III) speciation in a Eu(III)/PAHA/aAl2O3 ternary system. Colloids Surf. Physicochem. Eng. Asp. 435, 9-15.

Janot N., Reiller P. E., Zheng X., Croué J.-P. and Benedetti M. F. (2012) Characterization of humic acid reactivity modifications due to adsorption onto a-Al2O3. Water Res. 46, 731-740.

Kaiser K. (2003) Sorption of natural organic matter fractions to goethite ( $\alpha-\mathrm{FeOOH})$ : effect of chemical composition as revealed by liquid-state 13C NMR and wet-chemical analysis. Org. Geochem. 34, 1569-1579.

Kim S., Kramer R. W. and Hatcher P. G. (2003) Graphical Method for Analysis of UltrahighResolution Broadband Mass Spectra of Natural Organic Matter, the Van Krevelen Diagram. Anal. Chem. 75, 5336-5344.

Koch B. P. and Dittmar T. (2006) From mass to structure: an aromaticity index for high-resolution mass data of natural organic matter. Rapid Commun. Mass Spectrom. 20, 926-932.

Kujawinski E. B., Freitas M. A., Zang X., Hatcher P. G., Green-Church K. B. and Jones R. B. (2002) The application of electrospray ionization mass spectrometry (ESI MS) to the structural characterization of natural organic matter. Org. Geochem. 33, 171-180.

Kujawinski E. B., Hatcher P. G. and Freitas M. A. (2002) High-Resolution Fourier Transform Ion Cyclotron Resonance Mass Spectrometry of Humic and Fulvic Acids: Improvements and Comparisons. Anal. Chem. 74, 413-419.

Kummert R. and Stumm W. (1980) The surface complexation of organic acids on hydrous y-Al2O3. J. Colloid Interface Sci. 75, 373-385.

Leenheer J. A., Rostad C. E., Gates P. M., Furlong E. T. and Ferrer I. (2001) Molecular Resolution and Fragmentation of Fulvic Acid by Electrospray Ionization/Multistage Tandem Mass Spectrometry. Anal. Chem. 73, 1461-1471.

Loffredo E. and Senesi N. (2006) Fate of anthropogenic organic pollutants in soils with emphasis on adsorption/desorption processes of endocrine disruptor compounds. Pure Appl. Chem. $\mathbf{7 8 .}$

Meier M., Namjesnik-Dejanovic K., Maurice P. A., Chin Y.-P. and Aiken G. R. (1999) Fractionation of aquatic natural organic matter upon sorption to goethite and kaolinite. Chem. Geol. 157, 275-284.

Milne C. J., Kinniburgh D. G., Van Riemsdijk W. H. and Tipping E. (2003) Generic NICA-Donnan model parameters for metal-ion binding by humic substances. Environ. Sci. Technol. 37, 958-971.

Ochs M., Cosovic B. and Stumm W. (1994) Coordinative and hydrophobic interaction of humic substances with hydrophilic Al203 and hydrophobic mercury surfaces. Geochim. Cosmochim. Acta 58, 639-650.

Piccolo A. (2001) The supramolecular structure of humic substances. Soil Sci. 166, 810-832. 
Piccolo A., Conte P. and Cozzolino A. (2001) Chromatographic and spectrophotometric properties of dissolved humic substances compared with macromolecular polymers. Soil Sci. 166, 174185.

Piccolo A., Spiteller M. and Nebbioso A. (2010) Effects of sample properties and mass spectroscopic parameters on electrospray ionization mass spectra of size-fractions from a soil humic acid. Anal. Bioanal. Chem. 397, 3071-3078.

Plancque G., Amekraz B., Moulin V., Toulhoat P. and Moulin C. (2001) Molecular structure of fulvic acids by electrospray with quadrupole time-of-flight mass spectrometry. Rapid Commun. Mass Spectrom. 15, 827-835.

Pruett R. J. and Webb H. L. (1993) Sampling and analysis of KGa-1 B well-crystallized kaolin source clay. Clays Clay Miner. 41, 514-19.

Rebhun M., De Smedt F. and Rwetabula J. (1996) Dissolved humic substances for remediation of sites contaminated by organic pollutants. Binding-desorption model predictions. Water Res. 30, 2027-2038.

Reemtsma T. (2009) Determination of molecular formulas of natural organic matter molecules by (ultra-) high-resolution mass spectrometry. J. Chromatogr. A 1216, 3687-3701.

Reemtsma T., These A., Springer A. and Linscheid M. (2008) Differences in the molecular composition of fulvic acid size fractions detected by size-exclusion chromatography-on line Fourier transform ion cyclotron resonance (FTICR-) mass spectrometry. Water Res. 42, 6372.

Reiller P., Amekraz B. and Moulin C. (2006) Sorption of Aldrich humic acid onto hematite: Insights into fractionation phenomena by electrospray ionization with quadrupole time-of-flight mass spectrometry. Environ. Sci. Technol. 40, 2235-2241.

Rice J. A. and MacCarthy P. (1991) Statistical evaluation of the elemental composition of humic substances. Org. Geochem. 17, 635-648.

Ritchie J. D. and Perdue E. M. (2003) Proton-binding study of standard and reference fulvic acids, humic acids, and natural organic matter. Geochim. Cosmochim. Acta 67, 85-96.

Schlautman M. A. and Morgan J. J. (1994) Adsorption of aquatic humic substances on colloidal-size aluminum oxide particles: Influence of solution chemistry. Geochim. Cosmochim. Acta 58, 4293-4303.

Sparks D. L., Page A. L., Helmke P. A., Loeppert R. H. and Swift R. S. (1996) Organic Matter Characterization. In SSSA Book Series Soil Science Society of America, American Society of Agronomy.

Stenson A. C., Landing W. M., Marshall A. G. and Cooper W. T. (2002) Ionization and Fragmentation of Humic Substances in Electrospray Ionization Fourier Transform-lon Cyclotron Resonance Mass Spectrometry. Anal. Chem. 74, 4397-4409.

Stenson A. C., Marshall A. G. and Cooper W. T. (2003) Exact Masses and Chemical Formulas of Individual Suwannee River Fulvic Acids from Ultrahigh Resolution Electrospray lonization Fourier Transform lon Cyclotron Resonance Mass Spectra. Anal. Chem. 75, 1275-1284.

Sutton R. and Sposito G. (2005) Molecular Structure in Soil Humic Substances: The New View. Environ. Sci. Technol. 39, 9009-9015. 
Sverjensky D. A. and Sahai N. (1996) Thoretical prediction of single-site surface-protonation equilibrium constants for oxides and silicates in water. Geochim. Cosmochim. Acta 60, 3773-3797.

Swift R. S. (1989) Molecular weight, size, shape, and charge characteristics of humic substances: Some basic considerations. In Humic Substances II: In Search of Structure Wiley, Chichester, UK. pp. 449-466.

Tipping E. (2002) Cation binding by humic substances., Cambridge University Press, Cambridge, U.K.

Wang K. and Xing B. (2005) Structural and sorption characteristics of adsorbed humic acid on clay minerals. J. Environ. Qual. 34, 342-349.

Wershaw R. (1993) Model for humus in soils and sediments. Environ. Sci. Technol. 27, 814-816.

Wieland E. and Stumm W. (1992) Dissolution kinetics of kaolinite in acidic aqueous solutions at $25^{\circ} \mathrm{C}$. Geochim. Cosmochim. Acta 56, 3339-3355.

Yang S., Sheng G., Montavon G., Guo Z., Tan X., Grambow B. and Wang X. (2013) Investigation of $\mathrm{Eu}(\mathrm{III})$ immobilization on $\mathrm{y}$-Al2O3 surfaces by combining batch technique and EXAFS analyses: Role of contact time and humic acid. Geochim. Cosmochim. Acta 121, 84-104.

Zhou Q., Maurice P. A. and Cabaniss S. E. (2001) Size fractionation upon adsorption of fulvic acid on goethite: equilibrium and kinetic studies. Geochim. Cosmochim. Acta 65, 803-812. 